\title{
TO THE POSSIBILITY OF MEASUREMENTS OF ORTHOPOSITRONIUM ANNIHILATION AMPLITUDES FOR QED TESTING
}

\author{
V.G. Baryshevsky and O.N. Metelitsa \\ Institute of Nuclear Problems, Byelorussian State University \\ 11 Bobruiskaya Str., Minsk, 220050, Belarus
}

\begin{abstract}
In connection with previously revealed disagreement of theoretically calculated and measured orthopositronium decay rate in vacuum we suggest the experiment to clarify which of the positronium annililation amplitudes $R_{m}, m=0, \pm 1$ causes this disagreement. The developed for this purpose experimental procedure can also be used as the basis for the new area of positron spectroscopy, connected with the investigation of the anisotropic (spin dependent) interaction of positronium in the media.
\end{abstract}

PACS numbers: $36.10 . \mathrm{Dr}$

\section{Introduction}

Positronium atom (Ps) is an ideal object for testing of quantum electrodynamics (QED) of bound state (see review [1] and cited literature). It is caused by the particular role of hydrogen-like atoms which became traditional objects for the development of concepts and methods of quantum theory. Besides, there is no nucleus in Ps atom that is why this system can be calculated with any accuracy by QED. The measurements of orthopositronium decay rate, carried out in last ten years, have revealed the disagreement with the theory (0.2\% [2]). Different hypothesis as well as the assumption about the existence of mirror Universe [3] or about a new channel of positronium decay (photon + axion [4]) were proposed to explain this disagreement.

Ilowever, up to now the reasons of disagreement are not revealed. The understanding of the reasons of this disagreement requires detailed investigation of positronium decay, in particular, possible manifestation of anisotropic effects in this process.

In the positronium rest reference system the set of the three decay photons has the angular momentum equal to 1 and can have definite momentum projection to the quantization axis and to the normal of the decay plane simultaneously. (As well as molecule can have definite projection on the quantization axis and on the symmetry axis [5].) Therefore, the probability of polarized positronium decay on the three photons is described by three complex amplitudes $R_{m}$ [6], which correspond to $m=0, \pm 1$ projections of angular momentum to the normal of the 
decay plane. The difference in magnitude of the amplitudes $R_{m}$ will result in the anisotropy of angular distribution of decay photons. The difference in magnitude of one of the decay amplitudes in comparison with its theoretical value would result in the variation of orthopositronium decay rate compared to the theoretical prediction of QED. This difference can manifest itself also in the change of the angular distribution of three-photon decay of polarized positronium. In the present work we suggest the experiment allowing to investigate anisotropic phenomena under orthopositronium annihilation and to measure decay amplitudes $R_{m}$.

\section{Theoretical background}

It should be noted that the number of parameters which describe orthopositronium decay are less than six (three complex amplitudes). This is a result of the restriction imposed by invariance relative to $P_{-}, C P-, T$-transformations, and the rotational group and also by photons equivalence. The restrictions imposed by these symmetries have been theoretically investigated in Ref. [7]. The fulfillment of the $C_{-}, P_{-}, C P-, C P T$ - invariance has been verified in experiments [8-10]. The $P$ - (particularly $T$-) violation in positronium calculated on the basis of the Weinberg-Salam model [7] is much smaller than the modern experimental accuracy. Below let us assume the conditions imposed by these restrictions be fulfilled. In particular, this leads to the identity of angular distribution of photons produced by orthopositronium in $m= \pm 1$ states. Therefore, to observe the anisotropy of angular distributions of decay photons the population of the state with $m=0$ spin projection must differ from the average population of $m= \pm 1$ states. It means that we can use the aligned positronium.

The applications of polarized positrons in the fundamental theories testing involves several problems connected with a low accuracy of measurements of positron (and, therefore positronium) polarization in the matter $(\approx 10 \%[11])$. Hence, in proposed experiment we suggest to use unpolarized positron beam. The orthopositronium alignment required for the observation of anisotropic effects is produced by an external magnetic field. It is well known [12] that magnetic field reduces the lifetime of $m=0$ state of positronium but does not influence the $m= \pm 1$ states. The ratio of $m= \pm 1$ and $m=0$ state population is proportional to their lifetimes

$$
\frac{\rho_{00}}{\rho_{11}}=\frac{\rho_{00}}{\rho_{-1-1}}=\frac{\gamma_{q t}+\gamma_{\mathrm{p}}}{\gamma_{t}+\gamma_{\mathrm{p}}}, \quad \frac{\rho_{s s}}{\rho_{11}}=\frac{\gamma_{q s}+\gamma_{\mathrm{p}}}{\gamma_{t}+\gamma_{\mathrm{p}}},
$$

where $\rho_{11}$ and $\rho_{-1-1}$ are populations of $m= \pm 1$ states, correspondingly; $\rho_{00}, \gamma_{q t}$ and $\rho_{s s}, \gamma_{q s}$ are populations and decay rates of states transforming in the absence of magnetic field $B=0$, to the state with $m=0$ and singlet state, correspondingly, $\gamma_{\mathrm{p}}$ is the pick-off annihilation rate,

$$
\begin{aligned}
& \gamma_{q t}=C_{0}^{2} \gamma_{t}+C_{1}^{2} \gamma_{s}, \quad \gamma_{q s}=C_{1}^{2} \gamma_{t}+C_{0}^{2} \gamma_{s}, \\
& C_{0}^{2}=\frac{1}{2}\left[1+\left(1+x^{2}\right)^{-1 / 2}\right], \quad C_{1}^{2}=\frac{1}{2}\left[1-\left(1+x^{2}\right)^{-1 / 2}\right] .
\end{aligned}
$$

$C_{0}$ and $C_{1}$ are the coefficients of splitting of singlet and triplet positronium states [12], $x=4 \mu B / \Delta W=2.75 \times 10^{-3} B[\mathrm{~T}], \mu$ is the Bohr magneton, $\Delta W$ is the energy of the hyperfine splitting in a positronium atom. 
The annihilation amplitude squared $|T|^{2}$ of positronium in the state described by the spin density matrix $\rho$ can be written in the form

$$
\begin{aligned}
& |T|^{2}=\operatorname{Sp}(\rho Q), \\
& Q_{m n}=\langle 3 \gamma|\hat{T}| m\rangle\langle n|\hat{T}| 3 \gamma\rangle,
\end{aligned}
$$

where the amplitude $\langle 3 \gamma|\hat{T}| m\rangle$ describes three-photon decay of positronium in a stationary state $|m\rangle$.

It is best to analyze the spatial anisotropy of positronium decay in the Cartesian basis. In this basis the decay matrix $\boldsymbol{Q}$ is a symmetrical Cartesian tensor. Really, if the tensor $Q_{i k}$ had an antisymmetrical part equivalent to some vector, it would change sign in $P$ - $(T-)$ transformations [7], thus, the angular distribution of decay photons also would change.

QED-calculated squared amplitude of positronium annihilation is [13]:

$$
|M|^{2}=\frac{(8 \pi)^{3} e^{6}}{m^{2}} \operatorname{Sp}(\rho F) \text {. }
$$

$e, m$ are the electron charge and the mass correspondingly, the symmetrical tensor $F$ in the Cartesian basis has the form [13]:

$$
\begin{aligned}
F_{i k} & =\left(\delta_{i k}-n_{1 i} n_{1 k}\right)\left(1-n_{2} \cdot n_{3}\right)^{2}+\left(\delta_{i k}-n_{2 i} n_{2 k}\right)\left(1-n_{1} \cdot n_{3}\right)^{2} \\
& +\left(\delta_{i k}-n_{3 i} n_{3 k}\right)\left(1-n_{1} \cdot n_{2}\right)^{2},
\end{aligned}
$$

where $n_{l}$ is the unit vector along the momentum direction of $l$-th photon. A comparison of Eqs. (3), (4) and Eq. (2) shows their equivalence.

Substituting the density matrix (1) rewritten in the Cartesian basis to the expression for the annihilation amplitude (3) we obtain

$$
|M|^{2}=\frac{(8 \pi)^{3} e^{6}}{2 m^{2}}\left[\frac{F_{x x}+F_{y y}}{\gamma_{t}+\gamma_{\mathrm{p}}}+F_{z z}\left(\frac{C_{0}^{2}}{\gamma_{g t}+\gamma_{\mathrm{p}}}+\frac{C_{1}^{2}}{\gamma_{q s}+\gamma_{\mathrm{p}}}\right)\right] \text {. }
$$

Even although, it is necessary to use exact expression (5) for the measurement interpretation, we shall analyze this expression in the simple case of a weak magnetic field $x^{2} \ll 1$. Taking also into account that $\gamma_{t} \ll \gamma_{s}$ we obtain

$$
|M|^{2}=\frac{(4 \pi)^{3} e^{6}}{2 m^{2}}\left(\frac{F_{z z}}{\gamma_{t}+0.25 x^{2} \gamma_{s}}+\frac{F_{x x}+F_{y y}}{\gamma_{t}}\right) .
$$

It is significant that the restriction $x \ll 1$ does not influence the conclusion reached below.

According to (3) and (6) the angular distribution of decay photons is defined by the tensor $F$. The eigenvalues $\Lambda_{i}$ and the principal axes $\boldsymbol{e}_{i}$ of the symmetrical tensor $F$ depend only on the emission direction of photons $n_{i}, i=1,2,3$ :

$$
\begin{aligned}
\Lambda_{1}= & \frac{1}{2}(3 R-2 C), \quad \Lambda_{2}=\frac{1}{2}(-R+2 C), \quad \Lambda_{3}=\Lambda_{1}+\Lambda_{2}=R, \\
R= & \left(1-n_{1} \cdot n_{2}\right)^{2}+\left(1-n_{1} \cdot n_{3}\right)^{2}+\left(1-n_{2} \cdot n_{3}\right)^{2}, \\
C= & \left(1-n_{1} \cdot n_{2}\right)\left(1-n_{1} \cdot n_{3}\right)+\left(1-n_{1} \cdot n_{2}\right)\left(1-n_{2} \cdot n_{3}\right) \\
& +\left(1-n_{1} \cdot n_{3}\right)\left(1-n_{2} \cdot n_{3}\right) .
\end{aligned}
$$




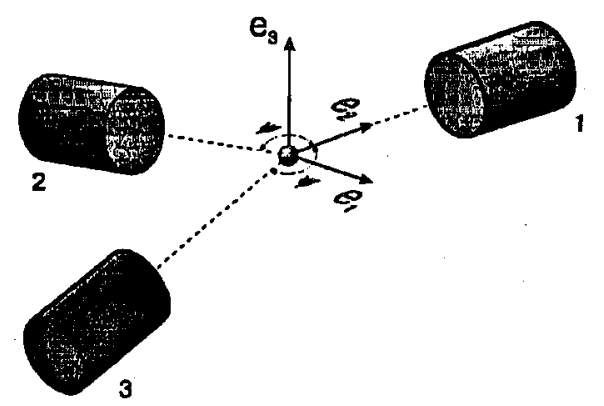

Fig. 1. The direction of the principal axes of the symmetrical tensor F (Eq. (4)) when the detectors 2, 3 are placed symmetrical relative to the detector 1 . The e2 axis is directed to center of detector 1 , the $\mathrm{e} 3$ axis is normal to the detector plane.

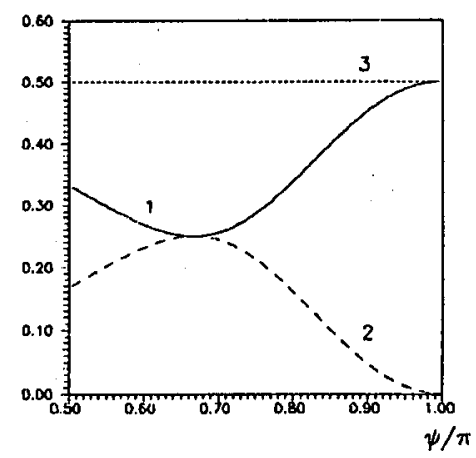

Fig. 2. A dependence of (1) $\Lambda_{1} /\left(\Lambda_{1}+\Lambda_{2}+\Lambda_{3}\right)$, (2) $\Lambda_{2} /\left(\Lambda_{1}+\Lambda_{2}+\Lambda_{3}\right)$, (3) $\Lambda_{2} /\left(\Lambda_{1}+\right.$ $\left.\Lambda_{2}+\Lambda_{3}\right)$, as a function of angle $\psi$ between the detectors.

If the detectors registering the three-photon decay are small, $\boldsymbol{n}_{i}$ coincide with the directions of the detector centers. In the special case of symmetrical position of detectors 2, 3 relative to the detector 1 the principal axis directions are shown in Fig. 1. The dependence of the eigenvalues on the angle $\psi$ between detectors is shown in Fig. 2.

It is necessary to explain the term of "anisotropy of angular distribution of decay photons formed by aligned positronium" before we start to describe the geometry of experiment. The decay anisotropy under consideration is not determined by the variation of coincidence count rate of detectors due to detectors displacement relative to each other on the plane, because the latter phenomenon also takes place for the nonaligned positronium. Here we consider the change of coincidence count rate of detectors as a function of the position of the detector plane as a whole relative to the external magnetic field direction.

\section{The geometry of the proposed experiment}

It can be shown that the cross-section (5), (6) and, therefore the coincidence count rate has an extremum if one of the principal axes $e_{i}$ of the tensor $F$ is 
directed along the magnetic field direction. If, for example, the $\boldsymbol{e}_{3}$-axis is directed along the magnetic field, we should substitute the following values in Eq. (5), (6):

$$
F_{z z}=\Lambda_{3}, \quad F_{x x}+F_{y y}=\Lambda_{1}+\Lambda_{2} .
$$

According to Eq. (5), (6) three eigenvalues of the tensor $F$ should be measured for the reconstruction of the decay photons angular distribution. We need to measure the coincidence rate only for three directions of the external magnetic field: $\boldsymbol{B}$ is directed along $\boldsymbol{e}_{i}, i=1,2,3$ (see Fig. 1). The positronium decay amplitudes can be easily calculated using well known expressions for the angular distribution of the products of the three-particle decay of the system with spin $S=1[6]$.

To suppress errors because of inhomogeneity of a magnetic field or inaccurate measurements of that we are going to measure the following quantity:

$$
\eta_{i k}=\frac{N_{i}-N_{0}}{N_{k}-N_{0}}=\frac{|M|_{i}^{2}-|M|_{0}^{2}}{|M|_{k}^{2}-|M|_{0}^{2}}, \quad i \neq k
$$

where $N_{i}$ and $|M|_{i}^{2}$ are the coincidence rate and squared annihilation amplitude for the magnetic field direction along $e_{i}$ (see Fig. 1), respectively, $N_{0}$ and $|M|_{0}^{2}$ are those in the absence of magnetic field $B=0$.

According to Eq. (6) the anisotropy degree $\eta_{i k}$ is determined by

$$
\eta_{i k}=\frac{\Lambda_{i}}{\Lambda_{k}} \text {. }
$$

First of all let us note that the numerator as well as the denominator of the expression (9) tend to zero if the magnetic field falls down. Therefore, although the anisotropy degree (10) does not depend on the magnetic field strength, the statistics being necessary for precision measurements $\eta_{i k}$ essentially decrease with the increase in the magnetic field.

Independence of anisotropy degree (10) on the pick-off annihilation rate and on the magnetic field strength (therefore, on the magnetic field inhomogeneity) permits to exclude corresponding systematic errors. As a result, in our experiment the errors caused by the presence of pick-off annihilation and, as is easy to see, by the presence of Van der Waals interactions of positronium, are much smaller than those in the decay rate measurements. Thus, the precision of measurement of anisotropy degree will be determined dominantly by the counting statistics.

By the contrast with pick-off annihilation, the ortho-para conversion may have an appreciable effect on the measurements of anisotropy degree because the ortho-para conversion changes the polarization and alignment of positronium in a magnetic field. This leads to the change in anisotropy degree. The equations describing the ortho-para conversion of positronium atom were considered in Refs. [14-17]. We do not conduct the solution of these equations in detail. The result is

$$
\eta_{i k}=\frac{\Lambda_{i}}{\Lambda_{k}}\left[1+\frac{\nu\left(\Lambda_{1}+\Lambda_{2}+\Lambda_{3}\right)}{\gamma_{t}+\gamma_{\mathrm{p}}}\left(\frac{1}{\Lambda_{i}}-\frac{1}{\Lambda_{k}}\right)\right],
$$

where $\nu$ is the ortho-para conversion rate. The expression (11) is obtained under the condition of $\nu \ll \gamma_{t}+\gamma_{p}, x^{2} \ll 1$. 
Thus, in the proposed experiments as well as in the measurements of ground states hyperfine splitting of positronium [18], the ortho-para conversion rate must be small and previously measured. On the other hand, the dependence of anisotropy degree on $\nu$ allows to use experimental procedure suggested in this work to investigate the ortho-para conversion process. In conclusion we note that proposed procedure is applicable for the investigation of anisotropy (spin dependent) interaction of positronium as well as for spin-exchange interaction with polarized media [17], and interaction with crystals and others.

The research described in this publication was made possible in part by grant No. MWU000 from the International Science Foundation.

\section{References}

[1] A. Rich, Rev. Mod. Phys. 53, 127 (1981).

[2] P. Hasbach, G. Hilkert, E. Klempt, G. Werth, Nuovo Cimento A 97, 419 (1987).

[3] S.L. Glashow, Phys. Lelt. B 167, 35 (1986).

[4] M.I. Dobroliubov, A.I. Ignatiev, Phys. Lett. B 229, 418 (1989).

[5] L.D. Landau, E.M. Lifshits, Quantum Mechanics, Nauka, Moskva 1989, p. 768.

[6] S.M. Berman, M. Jacob, Phys. Rev. B 139, 1023 (1965).

[7] W. Bernreuther, U. Low, O. Nachtmann, Hyperfine Interact. 44, 139 (1988).

[8] K. Marko, A. Rich, Phys. Rev. Lett. 33, 980 (1974).

[9] B.K. Arbic, S. Hatamian, M. Skalsey, J. Van House, W. Zheng, Phys. Rev. A 37, 3189 (1988).

[10] M. Skalsey, J. Van House, Phys. Rev. Lelt. 67, 1993 (1991).

[11] Y. Nagashima, T. Hyodo, Phys. Rev. B 41, 3937 (1990).

[12] V.I. Goldansky, Physical Chemistry of Positron and Positronium, Nauka, Moskva 1968.

[13] V.G. Baryshevsky, O.N. Metelitsa, V.V. Tichomirov, J. Phys. B, At. Mol. Phys. 22, 2835 (1989).

[14] A.P. Mills Jr., J. Chem. Phys. 62, 2646 (1975).

[15] A. Bisi, G. Consolati, G. Cambarini, L. Zappa, Nuovo Cimento B 65, 442 (1981).

[16] V.G. Baryshevsky, A.V. Ivashin, Zh. Exp. Teor. Fiz. 65, 1407 (1973).

[17] V.G. Baryshevsky, Physica Status Solidi B 124, 619 (1984).

[18] A.P. Mils, G.H. Bearman, Phys. Rev. Lett. 34, 246 (1975). 